\title{
TCL1A expression delineates biological and clinical variability in B-cell lymphoma
}

\author{
Mohit Aggarwal $^{1,4}$, Raquel Villuendas ${ }^{1}$, Gonzalo Gomez ${ }^{2}$, Socorro M Rodriguez-Pinilla ${ }^{1}$, \\ Margarita Sanchez-Beato ${ }^{1}$, David Alvarez ${ }^{1}$, Nerea Martinez ${ }^{1}$, Antonia Rodriguez ${ }^{1}$, \\ Maria E Castillo ${ }^{1}$, Francisca I Camacho ${ }^{1}$, Santiago Montes-Moreno ${ }^{1}$, Jose A Garcia-Marco ${ }^{3}$, \\ Eva Kimby ${ }^{4}$, David G Pisano ${ }^{2}$ and Miguel A Piris ${ }^{1}$ \\ ${ }^{1}$ Molecular Pathology Programme, Spanish National Cancer Research Centre (CNIO), Madrid, Spain; \\ ${ }^{2}$ Bioinformatics Unit, Structural Biology and Biocomputing Programme, CNIO, Madrid, Spain; ${ }^{3}$ Department \\ of Haematology, Hospital Universitario Puerta de Hierro, Madrid, Spain and ${ }^{4}$ Division of Hematology, \\ Department of Internal Medicine at Huddinge, Karolinska Institutet, Stockholm, Sweden
}

\begin{abstract}
The assembly of a collection of gene-expression signatures of the major types of B-cell non-Hodgkin's lymphoma has identified increased T-cell leukemia/lymphoma 1A (TCL1) expression in multiple lymphoma types and cases, and has enabled the investigation of the functional and clinical importance of TCL1 expression. Specifically, Burkitt's lymphoma cases show a homogeneously strong expression of TCL1, whereas diffuse large B-cell lymphoma, follicular lymphoma, mantle cell lymphoma, chronic lymphocytic leukemia, nodal marginal zone lymphoma, and splenic marginal zone lymphoma display a striking variability in the intensity of TCL1 staining. This was validated in two independent series. A Gene-Set Enrichment Analysis of the genes correlated with TCL1A expression found that variation in the level of expression of TCL1A was significantly associated with some of the most important gene signatures recognizing B-cell lymphoma pathogenesis and heterogeneity, such as germinal center, B-cell receptor, NF-кB (and its target genes), death, MAP kinases, TNFR1, TOLL, and IL1R. Additionally, TCL1 expression was correlated with shorter time to treatment in chronic lymphocytic leukemia cases and shorter lymphoma-specific survival in mantle cell lymphoma series, thus indicating the clinical and biological significance of TCL1 expression, and suggesting TCL1A as a potential therapeutic target.

Modern Pathology (2009) 22, 206-215; doi:10.1038/modpathol.2008.148; published online 26 September 2008
\end{abstract}

Keywords: TCL1; B-cell lymphoma; gene expression; clinical variability

Molecular pathogenesis of non-Hodgkin's lymphoma is determined by an interaction between the tumor cell and its microenvironment. ${ }^{1}$ This interaction controls the activation of B-cell receptor, NF- $\mathrm{BB}$, and other signaling pathways, and eventually regulates apoptosis and cell cycle., ${ }^{2,3}$ Some primary and secondary cytogenetic alterations have been identified that involve oncogenes such as $c-M Y C$ in Burkitt's lymphoma, BCL-2 translocation (t14;18) in follicular lymphoma, CCND1 translocation (t11;14) in mantle cell lymphoma, TCL1A deregulation in chronic lymphocytic leukemia, ${ }^{4}$ and $B C L-6$ deregulation in diffuse large B-cell lymphoma. ${ }^{5,6}$ However, there is an urgent need to identify additional accurate

Correspondence: Dr MA Piris, MD, PhD, Centro Nacional de Investigaciones Oncologicas, C/Melchor Fernandez Almagro no 3, Madrid E-28029, Spain.

E-mail: mapiris@cnio.es

Received 18 July 2008; revised and accepted 14 August 2008; published online 26 September 2008 molecular markers, and to apply our accumulated knowledge about lymphoma pathogenesis to targeted therapy. Although the effort expended on recognizing disease-specific molecular markers has led to the identification of many individual markers, such as CCND1, BCL6, ZAP70, and CD30, the molecular pathogenesis of the different types of non-Hodgkin's lymphoma seems to be characterized by the activation of some major B-cell lymphoma pathways that are commonly deregulated in B-cell non-Hodgkin's lymphoma types. To date, however, little has been done to identify nonlymphoma-type-specific alterations that could consistently determine lymphoma pathogenesis.

Here we have focused on the biological and clinical relevance of TCL1A expression, and its relation with $\mathrm{B}$-cell receptor activation. The involvement of B-cell receptor signaling ${ }^{7}$ and the TCL1A oncogene $^{8}$ in pathogenesis of B-cell non-Hodgkin's lymphoma has already been demonstrated. Dysregulation of TCL1A expression in T cells, by chromosome rearrangements, or in B cells, possibly 
by Epstein-Barr virus infection or microRNA targeting, enhances cell proliferation and survival, and leads to cell transformation following prolonged latency. ${ }^{4,9-11}$ Dysregulation of TCL1A, secondary to miR-29 and miR-181 loss, is a causal event in the pathogenesis of the aggressive form of chronic lymphocytic leukemia, and probably of splenic marginal zone lymphoma. ${ }^{9,12}$ Aberrant TCL1A expression also promotes malignant transformation of germinal center B cells. ${ }^{13}$ A role of TCL1A oncogene in the pathogenesis of other B-cell nonHodgkin's lymphoma malignancies has already been proposed, ${ }^{4}$ but the exact mechanisms by which TCL1A regulates tumor development in non-Hodgkin's lymphoma is not yet fully understood. ${ }^{14}$ Here we analyze gene-expression profiling data obtained from the analysis of the various B-cell lymphoma types, to investigate the functional significance of TCL1A in B-cell non-Hodgkin's lymphoma and the clinical relevance of increased TCL1A expression in mantle cell lymphoma and chronic lymphocytic leukemia.

\section{Materials and methods}

\section{Patients and Controls}

The microarray data (GSE9327) from 187 cases of B-cell non-Hodgkin's lymphoma available in the GEO public repository under MIAME regulations (http://www. ncbi.nlm.nih.gov/geo/query/acc.cgi? token $=$ ftenzyyykicqqfi\&acc $=$ GSE9327) were used in this study. The microarray data have been previously published by our group and include those for Burkitt's lymphoma (9 cases), diffuse large B-cell lymphoma (36 cases), ${ }^{15}$ mantle cell lymphoma (38 cases), ${ }^{16}$ chronic lymphocytic leukemia (38 cases), ${ }^{17}$ follicular lymphoma (33 cases), ${ }^{18}$ nodal marginal zone lymphoma (6), and splenic marginal zone lymphoma (27 cases). ${ }^{19}$ Reactive lymph nodes from eight patients and spleen from three patients were also analyzed. The cases were selected on the basis of the standard WHO Classification criteria. $^{20,21}$ All samples were fresh frozen diagnostic samples taken previous to treatment, and were provided by the hospitals and the CNIO Tumor Bank, under the supervision of the Local Ethic Committees.

In addition, an already published series of 98 cases of chronic lymphocytic leukemia hybridized on a chronic lymphocytic leukemia-specific $1.9 \mathrm{~K}$ Oligonucleotide Microarray (Agilent Technology) was used for TCL1A analysis. ${ }^{22}$ Main clinical characteristics of the patients ${ }^{22}$ are summarized in Table 1a.

Additional 64 patients diagnosed as mantle cell lymphoma ${ }^{23}$ were collected by the CNIO Tumor Bank Network under the supervision of the Local Ethic Committees. All cases were histologically reviewed by a team of pathologists (SMRP and $\mathrm{MAP}$ ), in accordance with the WHO criteria. ${ }^{20,21}$
Table 1 Main clinical findings from (a) CLL (98 patients) and (b) MCL (64 patients)

\begin{tabular}{|c|c|}
\hline Clinical variables & Total \\
\hline \multicolumn{2}{|l|}{ (a) $C L L$} \\
\hline \multicolumn{2}{|l|}{ Sex } \\
\hline Male & 60 \\
\hline Female & 38 \\
\hline \multicolumn{2}{|l|}{ Age at diagnosis (year) ${ }^{\mathrm{a}}$} \\
\hline$<60$ & 34 \\
\hline $60-69$ & 30 \\
\hline$\geqslant 70$ & 32 \\
\hline \multicolumn{2}{|l|}{ RAI stage } \\
\hline 0 & 52 \\
\hline$>0$ & 46 \\
\hline \multicolumn{2}{|l|}{ Binet stage } \\
\hline A & 76 \\
\hline $\mathrm{B}+\mathrm{C}$ & 22 \\
\hline \multicolumn{2}{|l|}{ CD38 expression ${ }^{\mathrm{a}}$} \\
\hline$<20 \%$ & 65 \\
\hline$\geqslant 20 \%$ & 23 \\
\hline \multicolumn{2}{|c|}{ First-line treatment (at 60 months of follow-up) } \\
\hline Yes & 43 \\
\hline No & 55 \\
\hline \multicolumn{2}{|l|}{ (b) $M C L$} \\
\hline \multicolumn{2}{|l|}{ Sex } \\
\hline Male & 45 \\
\hline Female & 19 \\
\hline \multicolumn{2}{|l|}{$\mathrm{Age}^{\mathrm{a}}$} \\
\hline$<60$ years & 21 \\
\hline$\geqslant 60$ years & 30 \\
\hline \multicolumn{2}{|l|}{ Stages $^{a}$} \\
\hline I & 0 \\
\hline II & 4 \\
\hline III & 7 \\
\hline IV & 48 \\
\hline \multicolumn{2}{|l|}{ Extranodal involvement ${ }^{\mathrm{a}}$} \\
\hline$<2$ & 39 \\
\hline$\geqslant 2$ & 25 \\
\hline \multicolumn{2}{|l|}{ Serum LDH ${ }^{\mathrm{a}}$} \\
\hline$\leqslant$ Normal & 38 \\
\hline$>$ Normal & 12 \\
\hline \multicolumn{2}{|l|}{ ECOG performance status ${ }^{\mathrm{a}}$} \\
\hline$<2$ & 42 \\
\hline$\geqslant 2$ & 11 \\
\hline \multicolumn{2}{|c|}{ International prognostic index ${ }^{a}$} \\
\hline Low/low-intermediate & 27 \\
\hline High/high-intermediate & 25 \\
\hline \multicolumn{2}{|c|}{ Overall survival (at 60 months) } \\
\hline Alive & 36 \\
\hline Dead & 28 \\
\hline
\end{tabular}

CLL, chronic lymphocytic leukemia; ECOG, Eastern Cooperative Oncology Group; LDH, lactate dehydrogenase; MCL, mantle cell lymphoma.

${ }^{\mathrm{a}}$ Data are not available for all the cases. 


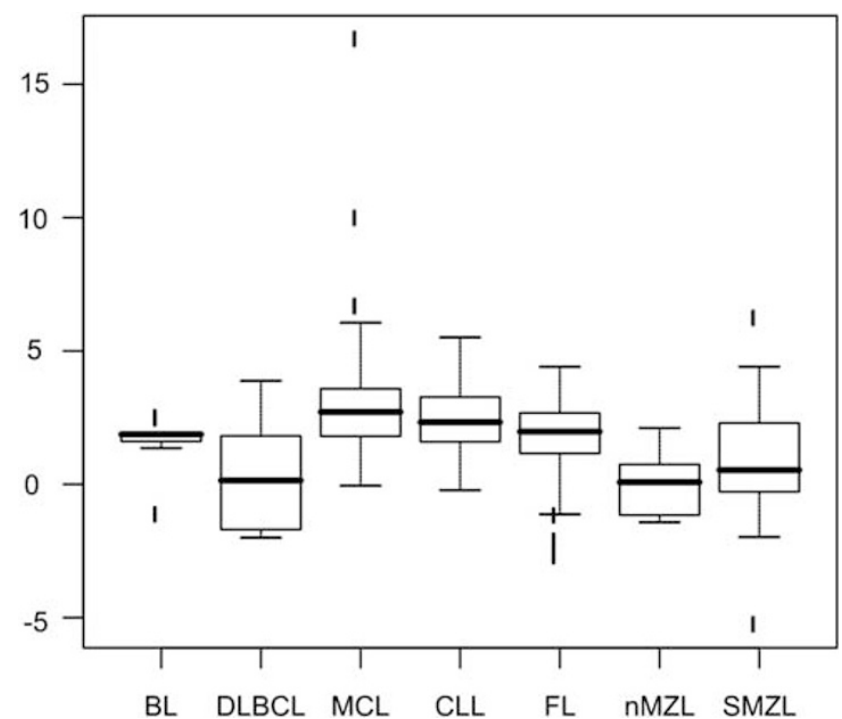

Figure 1 TCL1 gene expression in B-cell non-Hodgkin's lymphoma types: using cases hybridized on OncoChip. The expression for the cases was normalized with respect to the expression of reactive lymph nodes, whereas gene expression for splenic marginal zone lymphoma was normalized against normal spleen.

These samples were used to do a tissue microarray ${ }^{23}$ (see below). Tumoral diagnostic samples were obtained from untreated patients. Patient records and clinical data were reviewed retrospectively. The patient data for analyzable cases are summarized in Table $1 \mathrm{~b}$.

\section{Gene-Set Enrichment Analysis and Regulatory Network Analysis}

We conducted Gene-Set Enrichment Analysis to understand the functional pathways that are coregulated with the expression of TCL1A. The gene set database included Biocarta pathways, ${ }^{24}$ clusters of functionally related coregulated genes identified in unsupervised clustering by us, and molecular signatures defined for lymphoma in the Staudt molecular signature database (http://lymphochip. nih.gov/signaturedb). ${ }^{25}$ The gene sets that were coregulated with TCL1A expression were identified using Pearson's correlation, with a minimum of 15 genes in a gene set required to qualify for further analysis.

ARACNE was used to generate direct regulatory interactions with TCL1A; ${ }^{26}$ ARACNE is an algorithm that reverse engineers a gene regulatory network from microarray gene-expression data. The algorithm uses mutual information, an information theoretical measure, to compute the nonlinear correlation between pairs of genes and infer a bestfit network of probable interactions. The results show those best candidate genes $(P<0.05)$ that share a direct regulatory relationship with a particular gene target.

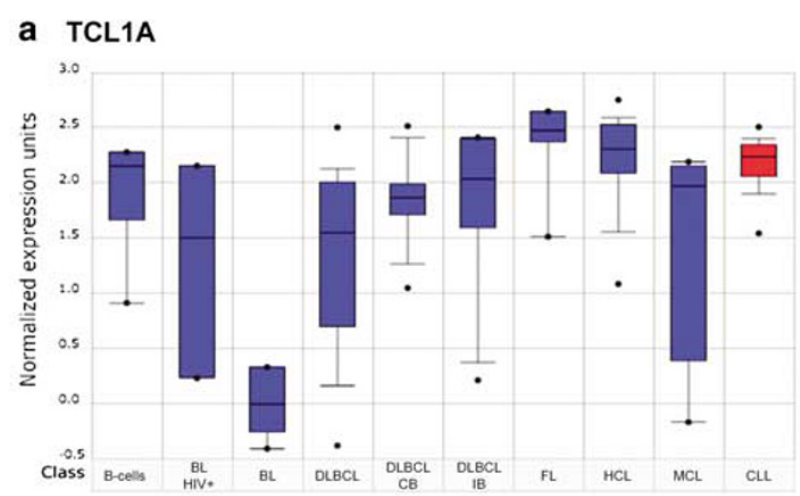

b TCL1A

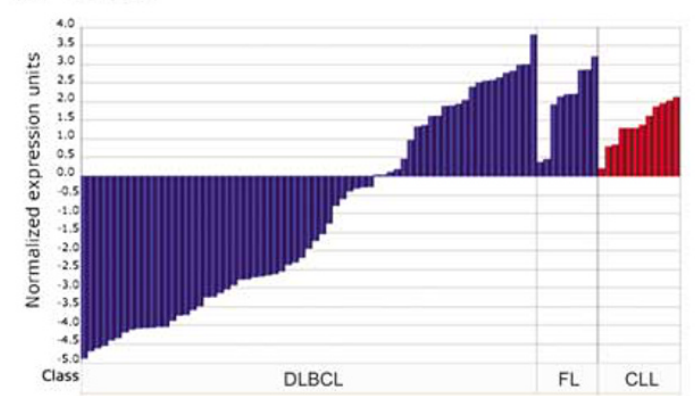

Figure 2 Oncomine analysis of TCL1A in non-Hodgkin's lymphoma: (a) Differential expression of TCL1A in lymphoma types: Burkitt's lymphoma (HIV +), diffuse large B-cell lymphoma (centroblastic), diffuse large B-cell lymphoma (immunoblastic), diffuse large B-cell lymphoma, hairy-cell Leukemia, Burkitt's lymphoma, follicular lymphoma, B-cells, mantle cell lymphoma, and chronic lymphocytic leukemia, respectively (www.oncomi ne.org). Data link source: http://www.ncbi.nlm.nih.gov/projects/ geo/query/acc.cgi?acc $=$ GSE2350. ${ }^{26}$ (b) TCL1A expression in diffuse large B-cell lymphoma, follicular lymphoma, and chronic lymphocytic leukemia. Data link sources: http://lmpp.nih.gov/ lymphoma/analysis.shtml, http://www.ncbi.nlm.nih.gov/geo/ query/acc.cgi?acc $=$ GSE60. ${ }^{35}$

\section{Tissue Microarray and Immunohistochemistry}

A tissue microarray ${ }^{23}$ with 64 cases of mantle cell lymphoma was stained with TCL1A (clone 27D6/20, MBL International, MA) and cyclin D1 antibodies (NeoMarkers-LabVision, Thermo Fisher Scientific Inc., Fremont, CA). The diagnosis of the cases was corroborated by positive cyclin D1 staining.

The expression of the studied markers was determined by the ARIOL semiautomated computerized training system (http://www.aicorp.com/ products/02path.htm). ${ }^{23}$ The system was trained by a team of technicians and pathologists to quantify TCL1A and CCND1 expression. The operation is similar to flow cytometry but on tissue sections, involving quantitative measurement (number of cells in a core of the patient's sample) and qualitative assessment (intensity of staining).

\section{Statistical Analysis}

Survival curves were produced by the Kaplan-Meier method. All deaths were attributed to the tumor. 


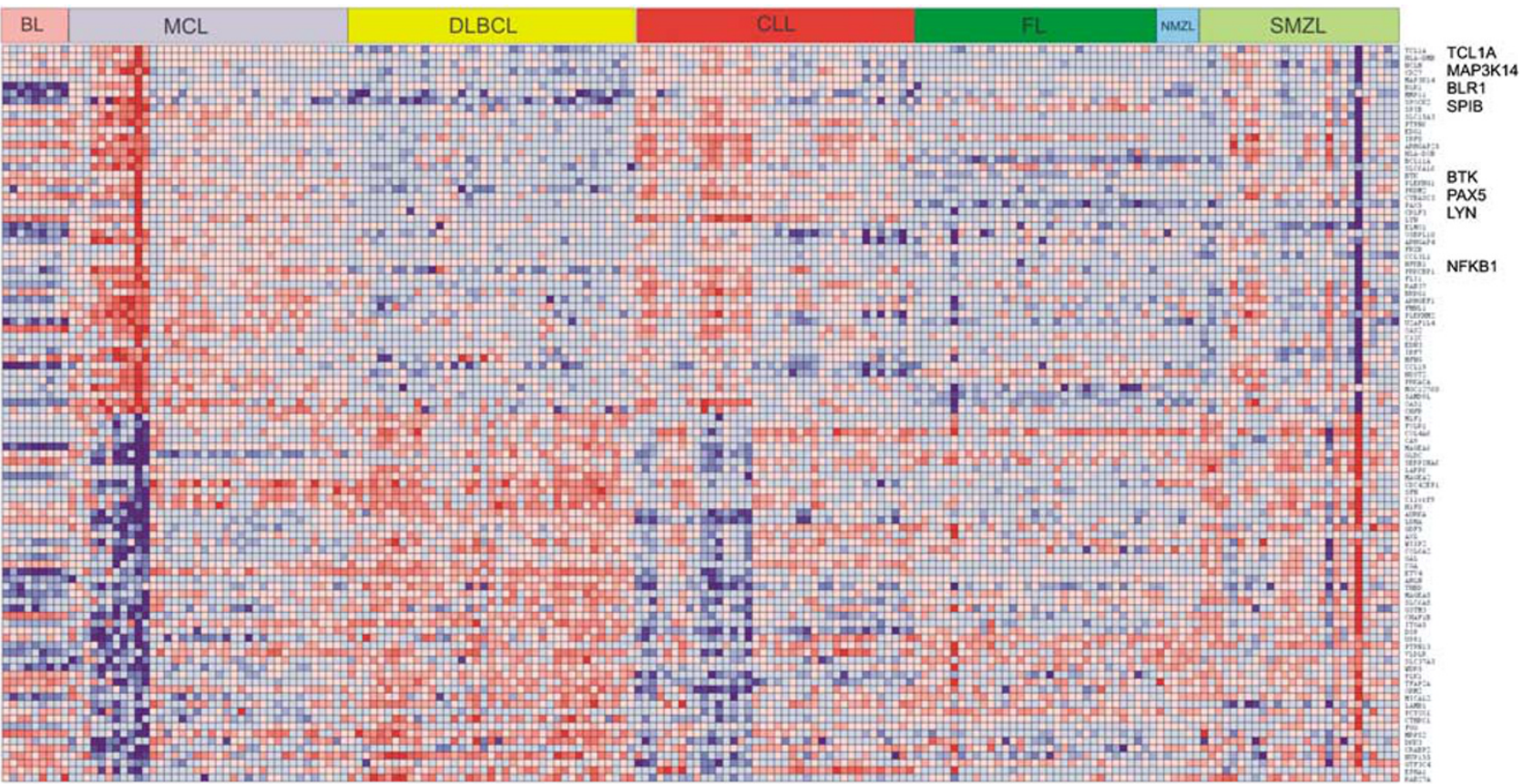

Figure 3 Heat map showing genes coregulated with variable expression of TCL1A in the B-cell non-Hodgkin's lymphoma series.

Table 2 Pathways coregulated with TCL1A expression in B-cell NHL: GSEA reporting on functionally relevant pathways that are statistically significant $(P<0.05, \mathrm{FDR}<25 \%)$ and report on positive Pearson's correlation with high level of expression of TCL1A

\begin{tabular}{|c|c|c|c|c|c|c|}
\hline Name of Gene-Set from GSEA & Size & $E S$ & $N E S$ & NOM p & $F D R q$ & Pathways \\
\hline CD40 signaling during GC dev & 91 & 0.53 & 1.94 & 0.00 & 0.03 & GC \\
\hline GC B cell & 90 & 0.39 & 1.52 & 0.06 & 0.12 & GC \\
\hline GC T-helper cell & 166 & 0.41 & 1.60 & 0.02 & 0.09 & GC \\
\hline Blood pan-B cell & 27 & 0.77 & 2.24 & 0.00 & 0.00 & BC \\
\hline SMZ B cell & 25 & 0.64 & 1.78 & 0.04 & 0.05 & BC \\
\hline BCR signal pathway & 23 & 0.51 & 1.68 & 0.04 & 0.06 & BC \\
\hline Toll pathway & 17 & 0.65 & 1.84 & 0.00 & 0.05 & BC \\
\hline NF-kB pathway & 16 & 0.69 & 1.73 & 0.00 & 0.05 & $N F-\kappa B$ \\
\hline $\mathrm{NF}-\kappa \mathrm{B}$ target genes & 18 & 0.52 & 1.31 & 0.20 & 0.21 & NF-кB \\
\hline TNFR2 pathway & 15 & 0.64 & 1.69 & 0.04 & 0.06 & NF-кB \\
\hline Death pathway & 23 & 0.43 & 1.33 & 0.13 & 0.19 & NF-кB \\
\hline IL1R pathway & 19 & 0.52 & 1.48 & 0.08 & 0.14 & $N F-\kappa B$ \\
\hline IL2 pathway & 18 & 0.49 & 1.46 & 0.13 & 0.15 & $N F-\kappa B$ \\
\hline IL2RB pathway & 27 & 0.43 & 1.37 & 0.10 & 0.17 & NF-кB \\
\hline TCR pathway & 30 & 0.49 & 1.67 & 0.02 & 0.06 & TC \\
\hline CD8 T-cell differentiation & 47 & 0.44 & 1.54 & 0.04 & 0.12 & TC \\
\hline CD4 T-cell differentiation & 78 & 0.40 & 1.53 & 0.00 & 0.12 & TC \\
\hline B-cell and T-cell calcium signaling & 76 & 0.43 & 1.44 & 0.05 & 0.15 & Calcium \\
\hline Blood NK cell & 18 & 0.49 & 1.45 & 0.10 & 0.14 & TC \\
\hline Resting dendritic cell & 30 & 0.45 & 1.40 & 0.10 & 0.16 & DC \\
\hline Plasmacytoid dendritic cell & 104 & 0.43 & 1.74 & 0.00 & 0.06 & DC \\
\hline CREB pathway & 18 & 0.43 & 1.29 & 0.19 & 0.21 & Calcium \\
\hline MAPK pathway & 59 & 0.37 & 1.48 & 0.04 & 0.14 & NF-кB, calcium, BC, TC \\
\hline BLIMP-1 targets & 88 & 0.48 & 1.66 & 0.07 & 0.05 & GC \\
\hline
\end{tabular}

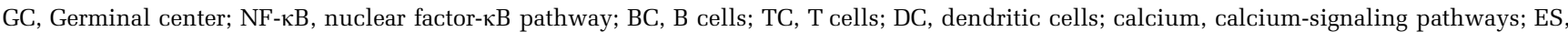
enrichment score; NES, normalized enrichment score; NOM p, nominal $P$-value; SMZ, splenic marginal zone lymphoma; BCR, B-cell receptor; TNFR, tumor-necrosis factor receptor; IL, interleukin; TCR, transcription-coupled repair; NK cells, natural killer cells; CREB, cAMP response element-binding protein; MAPK, mitogen-activated protein kinase; BLIMP-1, B lymphocyte-induced maturation protein-1; GSEA, Gene-Set Enrichment Analysis.

Statistical significance of associations between individual variables and survival was determined using the log-rank test. Pearson's correlation was applied to TCL1A and CCND1 expression analysis. All statistical analyses were performed using SPSS version 13.0 (SPSS Inc., Chicago, IL). 


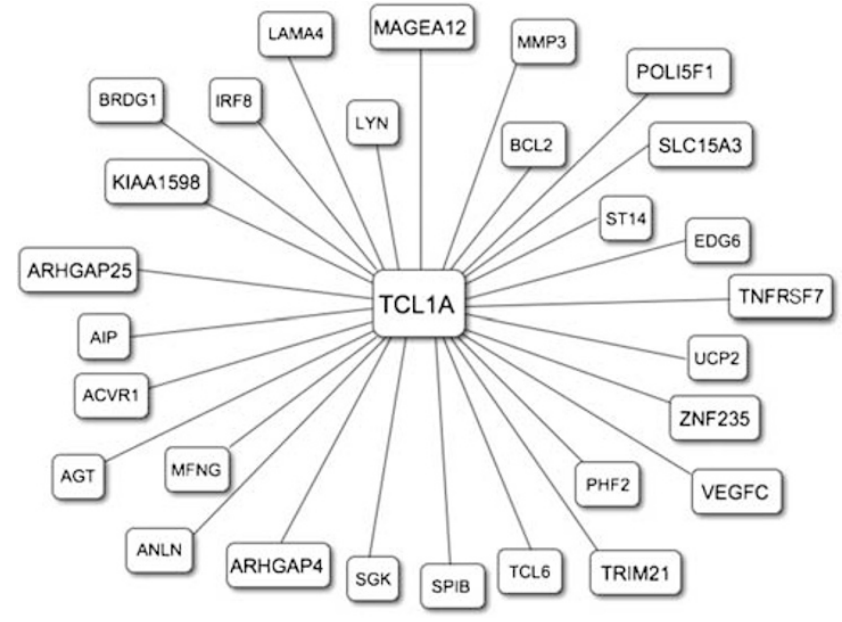

Figure 4 Network analysis for TCL1A in B-cell non-Hodgkin's lymphoma using ARACNE (Algorithm for the Reconstruction of Accurate Cellular Networks). The figure shows those best candidate genes $(P<0.05)$ that share a direct regulatory relationship with TCL1A.

\section{Results}

\section{TCL1A Expression in B-Cell Non-Hodgkin's Lymphoma}

The assembly of a collection of gene-expression signatures of the major types of B-cell non-Hodgkin's lymphoma enabled the functional and clinical relevance of genes presumably involved in the pathogenesis of specific tumor types to be investigated. An unexpected finding in our microarray series was the increased expression of TCL1A in different lymphoma types and cases (Figure 1), in spite of this gene expression being restricted to a subset of mantle zone cells in normal lymph nodes. Thus, Burkitt's lymphoma cases had a consistently strong expression of TCL1A, whereas diffuse large B-cell lymphoma, follicular lymphoma, mantle cell lymphoma, chronic lymphocytic leukemia, nodal marginal zone lymphoma, and splenic marginal zone lymphoma displayed striking variability in the intensity of TCL1A staining. This increased expression of TCL1A in different non-Hodgkin's lymphoma types and cases was validated by an Oncomine (www.oncomine.org) search from already published microarray data, where great variability in the intensity of TCL1A expression was noticed in most B-cell lymphoma types (Figure 2). To understand better the functional relevance of the increased expression of TCL1A, we performed an unsupervised hierarchical clustering of genes in the OncoChip $^{\mathrm{TM}}$ that showed the TCL1A oncogene to be coregulated in a cluster including B-cell receptorpathway genes, such as $B T K$ and $L Y N$, and genes involved in lymphoma pathogenesis (eg, POU2AF1 and BCL11A; Supplementary Figure 1), thus confirming our previous observations linking B-cell receptor genes with TCL1A expression in chronic lymphocytic leukemia, which were made using a different platform and series of cases. ${ }^{17}$ Hence, we have used gene-expression data to generate testable hypotheses about B-cell lymphoma pathogenesis.

Pathways coregulated with TCL1A across nonHodgkin's lymphoma types

Pearson's correlation identified the genes coregulated with the expression of TCL1A (Figure 3). It is of particular note that although increased microarray expression was seen in all the B-cell lymphoma series, it specifically appeared to establish distinct heterogeneity in chronic lymphocytic leukemia, mantle cell lymphoma, diffuse large B-cell lymphoma, follicular lymphoma, and splenic marginal zone lymphoma cases, whereby the groups featured either high or low levels of expression of TCL1A that were associated with changes in the expression of a large set of genes. To explore this in greater depth, we performed comparative Gene-Set Enrichment Analysis of the genes whose expression was correlated with TCL1A expression, and found that variation in the level of expression of TCL1A was significantly associated $(P<0.05)$ with some of the most important pathways controlling B-cell lymphoma pathogenesis and heterogeneity (Table 2). These pathways included those of the germinal center, B-cell receptor, NF- $\mathrm{KB}$ (and its target genes), death, MAP kinases, TNFR1, TOLL, and IL1R. The association of TCL1A with the expression of all these pathways implies that it may have a central role in B-cell lymphomagenesis. To explore further the genes that share a direct regulatory relationship with TCL1A, we applied ARACNE. Reverse engineering for TCL1A regulatory network confirmed most of the results obtained by Gene-Set Enrichment Analysis, including the genes LYN, BCL2, TNFRSF7, and TCL6 (Figure 4).

The functional relevance of TCL1A expression was validated by analyzing a previously published independent series of 98 chronic lymphocytic leukemia ${ }^{22}$ patients with Gene-Set Enrichment Analysis. Cases that strongly expressed TCL1A, more strongly expressed genes clustered in the gene sets identified as BLIMP-1 targets and the CREBP pathway, including important B-cell receptor signaling genes such as $S Y K, B T K$, and $A K T-1$. A low level of expression of TCL1A was associated with NF- $\kappa \mathrm{B}$ and its associated pathways, such as the CD40 signaling during GC development and IL1R pathway (Figure 5). TCL1A and ZAP70 expression were significantly correlated in this series $(P=0.007)$.

Clinical significance of TCL1A expression in chronic lymphocytic leukemia

B-cell receptors in TCL1A transgenic mice show very low level of somatic hypermutation, resembling those of aggressive human chronic lymphocytic leukemia with shorter time to treatment and poorer overall survival. ${ }^{10}$ Additionally, the expression of TCL1A in human chronic lymphocytic leukemia has 


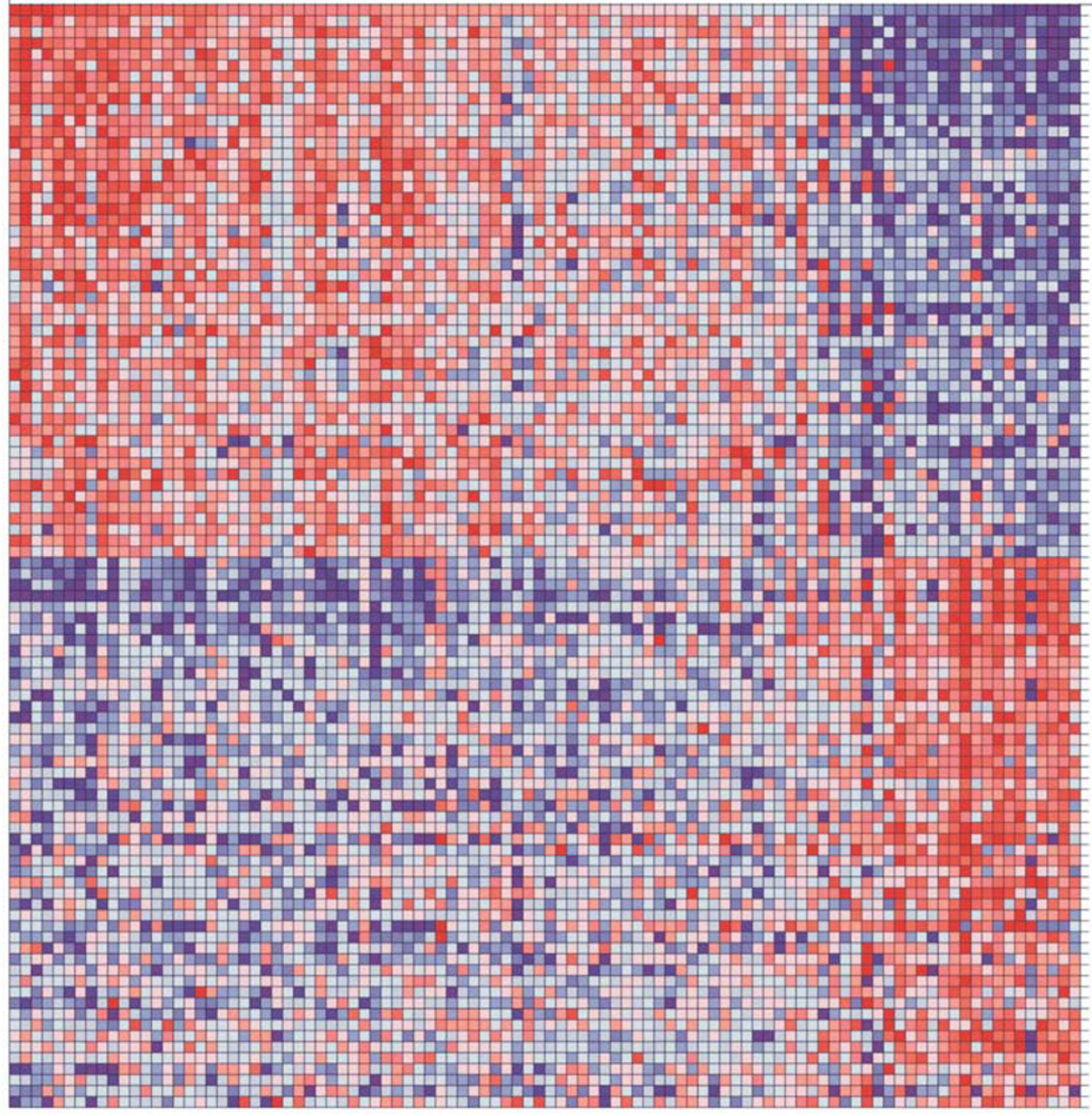

TCL1A
CD79B
TRAF5
TNFRSF7
BTK
IL2RG
SYK
TNFRSF17
IKBKB
TRADD
MAP2K2
CREB1
MAP4K2
PECAM1
PRKCB1
NFKB1L1
MAPK3
NFATC3
BAD
CARD4
Cr2

RIPK2
TNFAIP6
IL 6
BCL2A1
MAP3K8
MCL1
BCL6
NFKB1
IL1R1
PDK1
MAPK8
TNFAIP3
MAP3K2
IL1B
TNFSF9
RELB
REL
IL1ANFKBIE
NFKB2
PI3K
BIRC2
TRAF4
TNFSF10
TNS

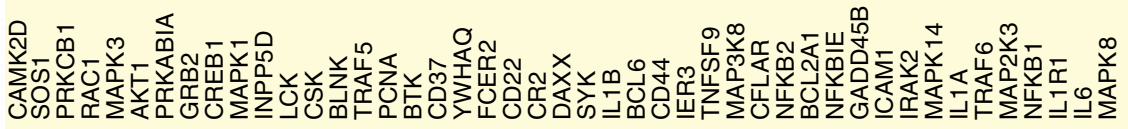

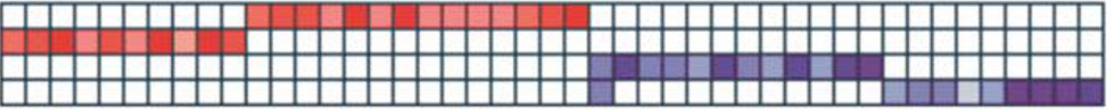

BLIMP-1 TARGETS
CREBP PATHWAY
CD40 SIGNALLING PATHWAY
IL1R PATHWAY

Figure 5 Heat map showing genes coregulated with variable expression of TCL1A oncogene in chronic lymphocytic leukemia (98 cases). Cases with stronger TCL1A expression show increased expression of coregulated pathways, such as B-cell receptor genes. Cases with weaker TCL1A expression have an NF-kB target-gene signature.

been associated with the presence of unmutated IgVH genes, ${ }^{11}$ but to the authors' knowledge, no direct proof of the clinical prognostic value of TCL1A expression in chronic lymphocytic leukemia has previously been published.

The analysis of the data generated by geneexpression profiling of an independent set of 98 chronic lymphocytic leukemia patients ${ }^{22}$ with a specific microarray platform shows that overexpression of TCL1A is associated with shorter time to treatment in chronic lymphocytic leukemia patients $(P<0.001$; Figure 6). In this analysis, the series was divided into four quartiles according to TCL1A expression, and cases in the lower quartile were compared with those in the other three quartiles.

Clinical significance of TCL1A expression in mantle cell lymphoma

A new series of 64 mantle cell lymphoma, ${ }^{23}$ cases was analyzed in a tissue microarray to establish 


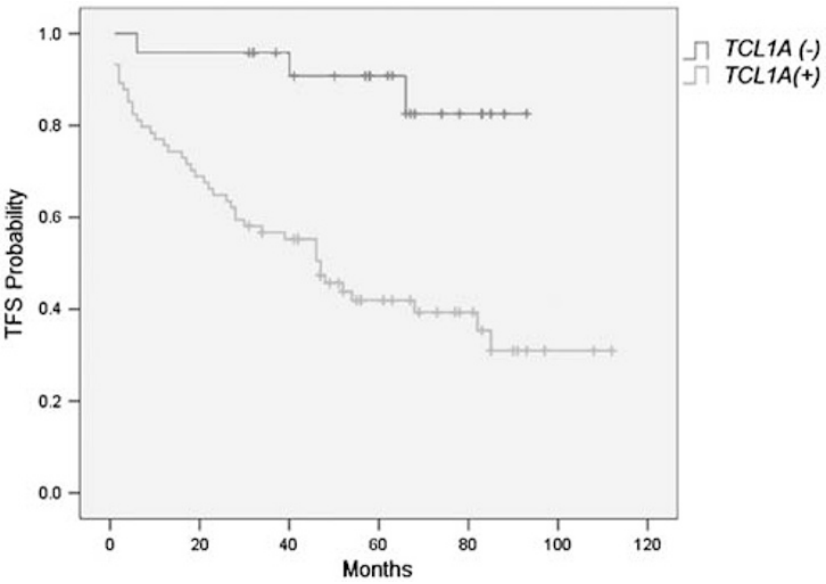

Figure 6 Prognostic significance of TCL1A oncogene in chronic lymphocytic leukemia: Kaplan-Meier analysis shows TCL1A microarray data in 98 cases of chronic lymphocytic leukemia (log-rank test $P$-value $<0.001)$. The cases were divided into quartiles of TCL1A expression and the lowest was compared to the top three quartiles. TFS: Treatment-free survival.

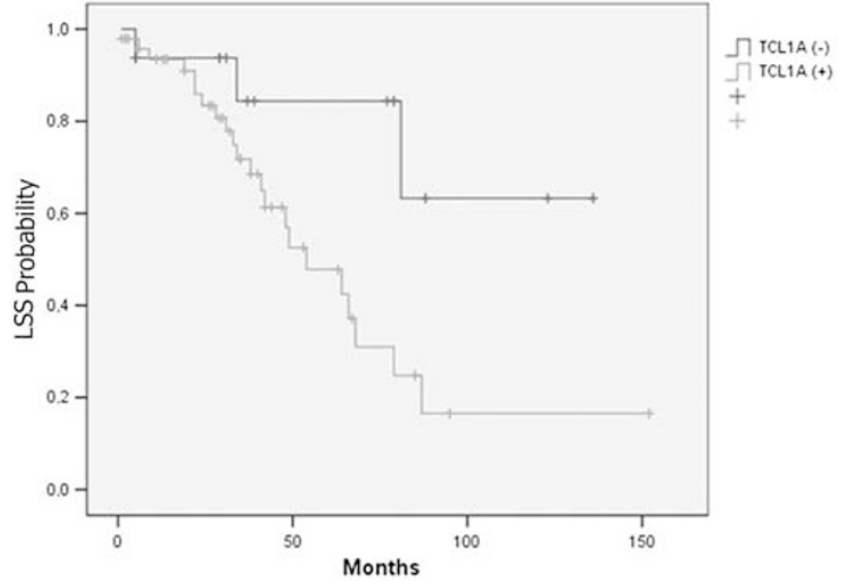

Figure 8 Prognostic value of TCL1A protein expression in mantle cell lymphoma: The Kaplan-Meier curve indicates better lymphoma-specific survival (LSS) for cases with weaker TCL1A expression in mantle cell lymphoma $(P=0.02)$. The cases were divided into quartiles of TCL1A expression and the lowest was compared to the top three quartiles.
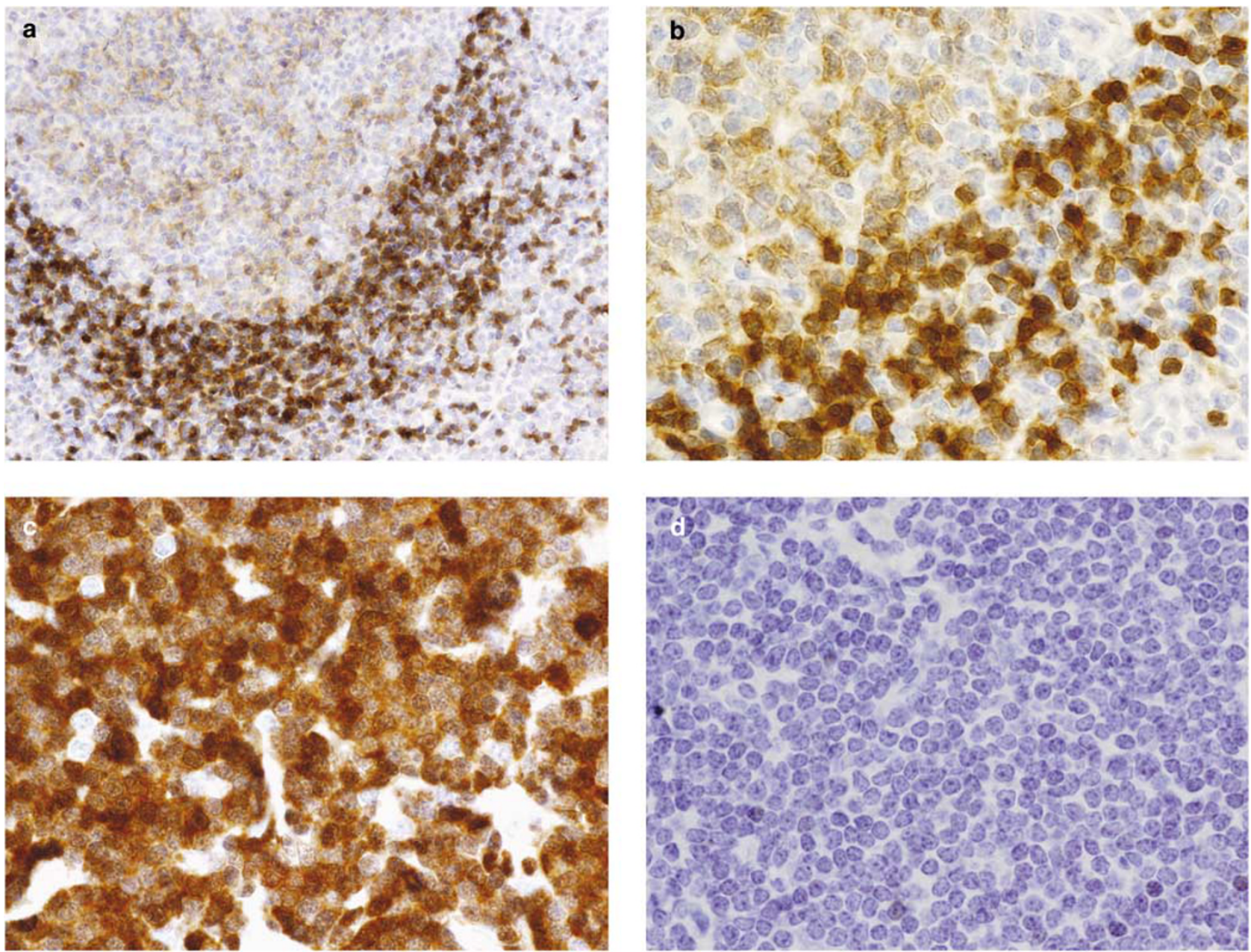

Figure 7 Expression of TCL1 in mantle cell lymphoma cases. (a and b) Expression of TCL1A in mantle zone cells in a reactive tonsil; (c) a mantle cell lymphoma case showing strong TCL1A expression; (d) an example of a mantle cell lymphoma case with TCL1A-negative expression. 
the clinical and biological significance of TCL1A protein expression (Figure 7). In this case, stronger TCL1A expression was associated with shorter survival probability $(P=0.02$; Figure 8$)$. For this analysis, the mantle cell lymphoma cases in the lowest quartile of TCL1A expression, which represents the $25 \%$ of cases with the lowest TCL1A intensity, were compared with those of the other three quartiles.

The correlation between TCL1A and cyclin D1 protein expression was also investigated. TCL1A and cyclin D1 expression were significantly correlated (Pearson's $R=0.283, P=0.024$ ). This same association was seen in the mRNA-expression array data of the Lymphochip (http://llmpp.nih.gov/; data not shown).

\section{Discussion}

Gene-expression profiling analysis of lymphomas has allowed collecting data from a large series of cases including all the major lymphoma types. One of the surprising findings of this study was the increased expression of TCL1A gene in multiple Bcell non-Hodgkin's lymphoma types, which confirms and extends previously published findings concerning precise lymphoma types. ${ }^{27}$

Physiological expression of TCL1A is largely limited to precursor/immature $\mathrm{T}$ and $\mathrm{B}$ lymphocytes and a subset of mantle zone B cells. ${ }^{11,27}$ However, lymphoma analysis shows that TCL1A expression is increased in specific cases or tumor types presumably deriving from TCL1A-negative cells subsets. Additionally, all tumor types, with the notable exception of Burkitt's lymphoma, show striking variability in the intensity of TCL1A expression. Although this had been previously described in specific lymphoma types, our observations indicate that increased TCL1A expression is a general phenomenon, occurring to some extent in all major B-cell lymphoma types. ${ }^{12,27,28}$ Interestingly, the only lymphoma type in which TCL1A expression was consistently high was Burkitt's lymphoma, a tumor whose normal counterpart is TCL1A-negative germinal center cells. Indeed TCL1A expression has been proposed as being a useful ancillary test of use in the diagnosis of Burkitt's lymphoma. ${ }^{29,30}$

The oncogenic capacity of TCL1A has been demonstrated by several groups. Thus, overexpression of the TCL1A gene in murine B-cells leads to a CD5(+) B-cell lymphoproliferative disorder with many of the features seen in the aggressive variant of human B-cell chronic lymphocytic leukemia, including a very low level of Ig somatic hypermutation. ${ }^{4,10}$ Hoyer and co-worker have also shown that dysregulated TCL1 promotes multiple classes of mature B-cell lymphoma. ${ }^{27}$

In this study, we have demonstrated an association between TCL1A expression and essential pathways for B-cell survival, including B-cell receptor, CD-40 signaling, NF-кB, TOLL, and calcium-signaling pathways. These findings seem to place TCL1A at the center of a crossroads that is essential for malignant B-cell survival, where B-cell apoptosis induced by antigen receptor crosslinking is blocked by a T-cell signal through CD $40 .^{31}$ This is consistent with the interpretation that the increased expression of TCL1A observed in multiple B-cell lymphoma types is the consequence of the escape from GC mechanisms of TCL1A repression. ${ }^{13}$

ARACNE generates a putative transcriptional network to predict broader functional relationships and could help in the understanding the biological role of TCL1A. One of the important findings of network analysis was probable regulatory interaction of TCL1A and CD27. It is known that TNFRSF7/CD27 binds to CD70, and plays a key role in regulating B-cell activation and immunoglobulin synthesis. It transduces signals that lead to the activation of NF$\kappa B$ and MAPK8/JNK. Adaptor proteins TRAF2 and TRAF5 have been shown to mediate the signaling process and are important in the apoptosis induced by TNFRSF7. TRAF1 and BCL2 are frequently found overexpressed in human chronic lymphocytic leukemia and this cooperation has been proposed to contribute to the development of chronic lymphocytic leukemia. ${ }^{32}$ Interestingly, the ARACNE study suggests a potential mechanism where TCL1A oncogene may provide a common link of functional regulation between B-cell receptor component (LYN), NF- $\mathrm{BB}$ pathway (pro-apoptotic signal via TNFRSF7), and survival pathway (BCL2) in B-cell non-Hodgkin's lymphoma.

We have also demonstrated the prognostic significance of TCL1A expression in chronic lymphocytic leukemia and mantle cell lymphoma in patients using RNA microarray and protein studies, respectively. This, together with the data showing that TCL1A expression is correlated with an adverse clinical outcome in diffuse large B-cell lymphoma ${ }^{33}$ is consistent with the proposed oncogenic role played by TCL1A expression. Gene-silencing studies may reveal how B-cell receptor and other coregulated pathways interact with the TCL1A oncogene at the molecular level.

Thus, these data contribute to the identification of lymphoma as the result of multiple oncogenic lesions and dysregulated pathways, some of the key drivers being B-cell receptor signaling and TCL1A expression acting via the calcium-signaling pathway. A potential therapeutic implication of these findings has been shown by Zanesi et $a{ }^{3}{ }^{34}$ where rapamycin inhibition of the Tcl1/Akt/mTOR pathway in $E \mu$-TCL1 transgenic mice prolonged the life of all treated animals.

\section{Acknowledgements}

This study was supported by grants from the Ministerio de Sanidad y Consumo (G03/179, 
PI051623, PI052800, CIBER-ER), the Ministerio de Ciencia y Tecnología (SAF2005-00221, SAF200404286, BIO 2005-01078), the Fundación la Caixa, Spain, and the National Institute of Bioinformatics (www.inab.org), a platform of Genoma Spain. This study was also supported by a Marie Curie $\mathrm{PhD}$ Early Stage Research Training fellowship. We appreciate the grammatical and linguistic review provided by Dr Philip Mason.

\section{Disclosure/conflict of interest}

The authors declare no conflicts of interest.

\section{References}

1 Herreros B, Sanchez-Aguilera A, Piris MA. Lymphoma microenvironment: culprit or innocent? Leukemia 2008;22:49-58.

2 Sanchez-Beato M, Sanchez-Aguilera A, Piris MA. Cell cycle deregulation in B-cell lymphomas. Blood 2003;101:1220-1235.

3 Staudt LM, Dave S. The biology of human lymphoid malignancies revealed by gene expression profiling. Adv Immunol 2005;87:163-208.

4 Bichi R, Shinton SA, Martin ES, et al. Human chronic lymphocytic leukemia modeled in mouse by targeted TCL1 expression. Proc Natl Acad Sci USA 2002;99:6955-6960.

5 Cattoretti G, Pasqualucci L, Ballon G, et al. Deregulated BCL6 expression recapitulates the pathogenesis of human diffuse large B cell lymphomas in mice. Cancer Cell 2005;7:445-455.

6 Polo JM, Juszczynski P, Monti S, et al. Transcriptional signature with differential expression of BCL6 target genes accurately identifies BCL6-dependent diffuse large $B$ cell lymphomas. Proc Natl Acad Sci USA 2007;104:3207-3212.

7 Kuppers R. Mechanisms of B-cell lymphoma pathogenesis. Nat Rev Cancer 2005;5:251-262.

8 Teitell MA. The TCL1 family of oncoproteins: co-activators of transformation. Nat Rev Cancer 2005;5:640-648.

9 Pekarsky Y, Santanam U, Cimmino A, et al. Tcl1 expression in chronic lymphocytic leukemia is regulated by miR-29 and miR-181. Cancer Res 2006;66:11590-11593.

10 Yan XJ, Albesiano E, Zanesi N, et al. B cell receptors in TCL1 transgenic mice resemble those of aggressive, treatment-resistant human chronic lymphocytic leukemia. Proc Natl Acad Sci USA 2006;103: 11713-11718.

11 Herling M, Patel KA, Khalili J, et al. TCL1 shows a regulated expression pattern in chronic lymphocytic leukemia that correlates with molecular subtypes and proliferative state. Leukemia 2006;20: 280-285.

12 Ruiz-Ballesteros E, Mollejo M, Mateo M, et al. MicroRNA losses in the frequently deleted region of $7 q$ in SMZL. Leukemia 2007;21:2547-2549.

13 Kuraishy AI, French SW, Sherman M, et al. TORC2 regulates germinal center repression of the TCL1 oncoprotein to promote B cell development and inhibit transformation. Proc Natl Acad Sci USA 2007;104:10175-10180.

14 Johnson AJ, Lucas DM, Muthusamy $\mathrm{N}$, et al. Characterization of the TCL- 1 transgenic mouse as a preclinical drug development tool for human chronic lymphocytic leukemia. Blood 2006;108: 1334-1338.

15 Tracey L, Perez-Rosado A, Artiga MJ, et al. Expression of the NF-kappaB targets BCL2 and BIRC5/Survivin characterizes small B-cell and aggressive B-cell lymphomas, respectively. J Pathol 2005;206:123-134.

16 Martinez N, Camacho FI, Algara P, et al. The molecular signature of mantle cell lymphoma reveals multiple signals favoring cell survival. Cancer Res 2003;63:8226-8232.

17 Rodriguez A, Martinez N, Camacho FI, et al. Variability in the degree of expression of phosphorylated IkappaBalpha in chronic lymphocytic leukemia cases with nodal involvement. Clin Cancer Res 2004;10: 6796-6806.

18 Ruiz-Vela A, Aggarwal $\mathrm{M}$, de la Cueva $\mathrm{P}$, et al. Lentiviral (HIV)-based RNA interference screen in human B-cell receptor regulatory networks reveals MCL1-induced oncogenic pathways. Blood 2008;111:1665-1676.

19 Ruiz-Ballesteros E, Mollejo M, Rodriguez A, et al. Splenic marginal zone lymphoma: proposal of new diagnostic and prognostic markers identified after tissue and cDNA microarray analysis. Blood 2005;106:1831-1838.

20 Harris NL, Jaffe ES, Stein H, et al. A revised EuropeanAmerican classification of lymphoid neoplasms: a proposal from the International Lymphoma Study Group. Blood 1994;84:1361-1392.

21 Jaffe ES, World Health Organization. Pathology and Genetics of Tumours of Haematopoietic and Lymphoid Tissues. IARC Press; Oxford University Press (distributor): Lyon; Oxford, 2001.

22 Rodriguez A, Villuendas R, Yanez L, et al. Molecular heterogeneity in chronic lymphocytic leukemia is dependent on BCR signaling: clinical correlation. Leukemia 2007;21:1984-1991.

23 Tracey L, Aggarwal M, Garcia-Cosio M, et al. Somatic hypermutation signature in B-cell low-grade lymphomas. Haematologica 2008;93:1186-1194.

24 Wei G, Twomey D, Lamb J, et al. Gene expressionbased chemical genomics identifies rapamycin as a modulator of MCL1 and glucocorticoid resistance. Cancer Cell 2006;10:331-342.

25 Shaffer AL, Wright G, Yang L, et al. A library of gene expression signatures to illuminate normal and pathological lymphoid biology. Immunol Rev 2006;210: 67-85.

26 Basso K, Margolin AA, Stolovitzky G, et al. Reverse engineering of regulatory networks in human B cells. Nat Genet 2005;37:382-390.

27 Said JW, Hoyer KK, French SW, et al. TCL1 oncogene expression in B cell subsets from lymphoid hyperplasia and distinct classes of B cell lymphoma. Lab Invest 2001;81:555-564.

28 Narducci MG, Pescarmona E, Lazzeri C, et al. Regulation of TCL1 expression in B- and T-cell lymphomas and reactive lymphoid tissues. Cancer Res 2000;60:2095-2100.

29 Harris NL, Horning SJ. Burkitt's lymphoma-the message from microarrays. N Engl J Med 2006; 354:2495-2498. 
30 Rodig SJ, Vergilio JA, Shahsafaei A, et al. Characteristic expression patterns of TCL1, CD38, and CD44 identify aggressive lymphomas harboring a MYC translocation. Am J Surg Pathol 2008;32:113-122.

31 Tsubata T, Wu J, Honjo T. B-cell apoptosis induced by antigen receptor crosslinking is blocked by a T-cell signal through CD40. Nature 1993;364:645-648.

32 Zapata JM, Krajewska M, Morse III HC, et al. TNF receptor-associated factor (TRAF) domain and Bcl-2 cooperate to induce small B cell lymphoma/chronic lymphocytic leukemia in transgenic mice. Proc Natl Acad Sci USA 2004;101:16600-16605.
33 Ramuz O, Bouabdallah R, Devilard E, et al. Identification of TCL1A as an immunohistochemical marker of adverse outcome in diffuse large B-cell lymphomas. Int J Oncol 2005;26:151-157.

34 Zanesi N, Aqeilan R, Drusco A, et al. Effect of rapamycin on mouse chronic lymphocytic leukemia and the development of nonhematopoietic malignancies in Emu-TCL1 transgenic mice. Cancer Res 2006;66:915-920.

35 Alizadeh AA, Eisen MB, Davis RE, et al. Distinct types of diffuse large B-cell lymphoma identified by gene expression profiling. Nature 2000;403:503-511.

Supplementary Information accompanies the paper on Modern Pathology website (http://www.nature.com/ modpathol) 